\title{
Non Markovian Queuing System with Restricted Admissibility Method
}

\author{
S. Maragathasundari, R. S. Somasundaram, S. Radha
}

\begin{abstract}
This article take a glance at a cluster area single server channel Queuing system, where the server gives two sorts of vacations viz., beginning one a short vacation and the long vacation is permitted as a second vacation. Long vacation is given in two stages. First stage is compulsory and if in case of need, the server goes for a optional second stage of long vacation. In addition, the concept of restricted admissibility of customers to the system is applied during the time of optional second stage of long vacation. For the above outlined covering issue, the beneficial variable method and probability approach are utilized to determine the Probability generating capacity of the line measure and the normal length of the line. In like way the other execution degrees of the model are settled utilizing Little's law. At last, the model is guarded by procedures for numerical redirection and the model is top notch by the graphical examination.
\end{abstract}

Keywords: Single arrival, Compulsory long vacation, Optional service, Restricted admissibility

\section{INTRODUCTION}

"Reference [6] derived the steady state queue size distribution at a random point of time as well as at a departure epoch". "Reference [4] made a study on extended vacation and service interruption". "Reference [9] examined balking and re-service in a vacation queue with batch arrival and two types of heterogeneous service". "Reference [5] analyzed mobile adhoc networks problem- A queuing approach". "Reference [7] analyzed multiple server vacation". "Reference [8] investigated queue with breakdown and reneging". A study on the analysis of performance measure of bulk input queue with $\mathrm{N}$ type of additional optional service, service interruption and deterministic vacation examined by [11]. "Reference [3] investigated a queuing model on FIFO basis". "Reference [1] investigated the Queuing process". "Reference [13] examined some new results for departure process in the $M^{X} / G / 1$ queuing system with a single vacation and exhaustive service". "Reference [2] has been studied restricted admissibility on Queues".

Revised Manuscript Received on December 15, 2019

* Correspondence Author

S. Maragatha Sundari*, Department of Mathematics, Kalasalingam Academy of Research and Education, Krishnankovil, Tamilnadu. Email: maragatham01@gmail.com

R. S. Somasundaram, Department of Computer applications, Coimbatore Institute of

Technology,Coimbatore-641014,Tanukbadu,Email:somasundram73@gmai 1.com

S. Radha, Department of Mathematics, Kalasalingam Academy of Research and Education, Krishnankovil, Tamilnadu. Email: kradhai87@gmail.com

\section{MATHEMATICAL DESCRIPTION OF THE QUEUEING MODEL}

The arithmetical interpretation of the Queuing framework has the capacity to be described by the resulting hypothesis:

Clients touch base at the framework in clumps of variable size in a compound procedure follows Poisson distribution. The administration time pursues general (arbitrary) circulation. First stage of giving service follows distribution function as $\overline{F_{1}}(x)$ and density function $\bar{f}_{1}(x)$. Let $\gamma_{r}(x) d x$ be the conditional density function. Hence we have

$$
\gamma_{r}(x)=\frac{\overline{f_{1}}(x)}{1-\overline{F_{1}}(x)}, \overline{f_{1}}(x)=\gamma_{r}(x) e^{-\int_{0}^{s} \gamma_{r}(s) d s}
$$

For short vacation,

$$
\gamma_{s}(x)=\frac{\overline{f_{2}(x)}}{1-\bar{F}_{2}(x)} \quad, \bar{f}_{2}(x)=\gamma_{s}(x) e^{-\int_{0}^{s} \gamma_{s}(s) d s}
$$

(b)

For compulsory long vacation,

$$
\gamma_{l_{1}}(x)=\frac{\overline{f_{3}}(x)}{1-\overline{F_{2}}(x)} \quad, \overline{f_{3}}(x)=\gamma_{l_{1}}(x) e^{-\int_{0}^{s} \gamma_{l_{1}}(s) d s}
$$

(c)

For optional long vacation,

$$
\gamma_{l_{2}}(x)=\frac{\overline{f_{4}}(x)}{1-\overline{F_{4}}(x)} \quad, \overline{f_{4}}(x)=\gamma_{l_{2}}(x) e^{-\int_{0}^{s} \gamma_{l_{2}}(s) d s}
$$

(d)

Restricted admissibility occurs with mean rate $\beta>0$.

\section{GOVERNING EQUATIONS OF THE MODEL}

$\frac{\partial}{\partial x} R_{n}(x)+\left(\lambda^{r} \gamma_{r}(x)\right) R_{n}(x)=\lambda^{r} R_{n-1}(x)$

$\frac{\partial}{\partial x} R_{0}(x)+\left(\lambda^{r} \gamma_{r}(x)\right) R_{0}(x)=0$

$\partial x x_{0}(x)+\left(\lambda^{r} \gamma_{r}(x)\right) R_{0}(x)=0$

$\frac{\partial}{\partial x} S_{n}(x)+\left(\lambda^{r}+\gamma_{s}(x)\right) S_{n}(x)=\lambda^{r} S_{n-1}(x)$

$\frac{\partial}{\partial x} S_{0}(x)+\left(\lambda^{r}+\gamma_{s}(x)\right) S_{0}(x)=0$

$\frac{\partial}{\partial x} L_{n}^{(1)}(x)+\left(\lambda^{r}+\gamma_{l_{1}}(x)\right) L_{n}^{(1)}(x)=\lambda^{r} L_{n-1}^{(1)}(x)$

$\frac{\partial}{\partial x} L_{0}^{(1)}(x)+\left(\lambda^{r}+\gamma_{l_{1}}(x)\right) L_{0}^{(1)}(x)=0$

$\frac{\partial}{\partial x} L_{n}^{(2)}(x)+\left(\lambda^{r}+\gamma_{l_{2}}(x)\right) L_{n}^{(2)}(x)=\lambda^{r} \beta L_{n-1}^{(2)}(x)+$

$\lambda^{r}(1-\beta) L_{n}^{(2)}(x)$

$\frac{\partial}{\partial x} L_{0}^{(2)}(x)+\left(\lambda^{r}+\gamma_{l_{2}}(x)\right) L_{0}^{(2)}(x)=\lambda^{r}(1-\beta) L_{0}^{(2)}(x)$

(8)

$\lambda k=$

$$
\begin{aligned}
& (1-c) \int_{0}^{\infty} R_{0}(x) \gamma_{r}(x) d x+(1-p) \int_{0}^{\infty} S_{0}(x) \gamma_{s}(x) d x+ \\
& (1-q) \int_{0}^{\infty} L_{0}^{(1)}(x) \gamma_{l_{1}}(x) d x+\int_{0}^{\infty} L_{0}^{(2)}(x) \gamma_{l_{2}}(x) d x
\end{aligned}
$$

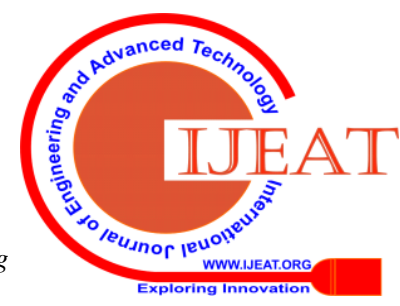




\section{BOUNDARY CONDITIONS}

The following boundary conditions are used to solve the above equations:

$$
\begin{aligned}
& R_{n}(0)= \\
& (1-c) \int_{0}^{\infty} R_{n-1}(x) \gamma_{r}(x) d x+ \\
& (1-p) \int_{0}^{\infty} S_{n+1}(x) \gamma_{s}(x) d x+ \\
& (1-q) \int_{0}^{\infty} L_{n+1}^{(1)}(x) \gamma_{l_{1}}(x) d x+\int_{0}^{\infty} L_{n+2}^{(2)}(x) \gamma_{l_{2}}(x) d x+\lambda k
\end{aligned}
$$$$
S_{n}(0)=c \int_{0}^{\infty} R_{n}(x) \gamma_{r}(x) d x
$$$$
L_{n}^{(1)}(0)=p \int_{0}^{\infty} S_{n}(x) \gamma_{s}(x) d x
$$$$
L_{n}^{(2)}=q \int_{0}^{\infty} L_{n}^{(1)}(x) \gamma_{l_{1}}(x) d x
$$$$
\sum_{n=1}^{\infty}(1) X z^{n}+(2) \text { gives }
$$$$
\frac{\partial}{\partial x} R_{d}(x, z)+\left(\lambda^{r}-\lambda^{r} z+\gamma_{r}(x)\right) R_{d}(x, z)=0
$$

(14)

Similarly,

$\frac{\partial}{\partial x} S_{d}(x, z)+\left(\lambda^{r}-\lambda^{r} z+\gamma_{s}(x)\right) S_{d}(x, z)=0$

(15)

$$
\begin{aligned}
& \frac{\partial}{\partial x} L_{d}^{(1)}(x, z)+\left(\lambda^{r}-\lambda^{r} z+\gamma_{l_{1}}(x)\right) L_{d}^{(1)}(x, z)=0 \\
& \frac{\partial}{\partial x} L_{d}^{(2)}(x, z)+\left(\lambda^{r} \beta-\lambda^{r} \beta z+\gamma_{l_{2}}(x)\right) L_{d}^{(2)}(x, z)=0
\end{aligned}
$$

Applying the same for boundary conditions, we get $z R_{d}(0, z)=(1-c) \int_{0}^{\infty} R_{d}(x, z) \gamma_{r}(x) d x+(1-$ p) $\int_{0}^{\infty} S_{d}(x, z) \gamma_{s}(x) d x+(1-q) \int_{0}^{\infty} L_{d}^{(1)}(x, z) \gamma_{l_{1}}(x) d x+$ $\int_{0}^{\infty} L_{d}^{(2)}(x, z) \gamma_{l_{2}}(x) d x+\lambda z k-\lambda k$

$S_{d}(0, z)=c \int_{0}^{\infty} R_{d}(x, z) \gamma_{r}(x) d x$

$L_{d}^{(1)}(0, z)=p \int_{0}^{\infty} S_{d}(x, z) \gamma_{s}(x) d x$

$L_{d}^{(2)}(0, z)=q \int_{0}^{\infty} L_{d}^{(1)}(x, z) \gamma_{l_{1}}(x) d x$

(14) from 0 to $x$ gives

$$
R_{d}(x, z)=R_{d}(0, z) e^{-\left(\lambda^{r}-\lambda^{r} z\right) x-\int_{0}^{x} \gamma_{r}(t) d t}
$$

Integrating (22) by parts we get

$R_{d}(z)=R_{d}(0, z)\left(\frac{1-\overline{F_{1}}(h)}{h}\right)$

Where $h=\lambda^{r}-\lambda^{r} z$

Multiply (22) by $\gamma_{r}(x) d x$ and integrating by parts, we get $\int_{0}^{\infty} R_{d}(x, z) \gamma_{r}(x) d x=R_{d}(0, z) \bar{F}_{1}(h)$

Similarly, we have

$$
\begin{aligned}
& S_{d}(z)=c R_{d}(0, z) \bar{F}_{1}(h)\left(\frac{1-\overline{F_{2}}(h)}{h}\right) \\
& \int_{0}^{\infty} S_{d}(x, z) \gamma_{s}(x) d x=c R_{d}(0, z) \overline{F_{1}}(h) \overline{F_{2}}(h) \\
& L_{d}^{(1)}(z)=p c R_{d}(0, z) \overline{F_{1}}(h) \overline{F_{2}}(h)\left(\frac{1-\overline{F_{3}}(h)}{h}\right) \\
& \int_{0}^{\infty} L_{d}^{(1)}(x, z) \gamma_{l_{1}}(x) d x=p c R_{d}(0, z) \overline{F_{1}}(h) \overline{F_{2}}(h) \overline{F_{3}}(h) \\
& L_{d}^{(2)}(z)=q p c R_{d}(0, z) \overline{F_{1}}(h) \overline{F_{2}}(h) \overline{F_{3}}(h)\left(\frac{1-\overline{F_{4}}(e)}{h}\right) \\
& \text { Where } e=\lambda^{r} \beta+\lambda^{r} \beta z
\end{aligned}
$$

$$
\int_{0}^{\infty} L_{d}^{(2)}(x, z) \gamma_{l_{2}}(x) d x=q p c R_{d}(0, z) \bar{F}_{1}(h) \overline{F_{2}}(h) \overline{F_{3}}(h) \bar{F}_{4}(e)
$$

Substituting (24), (26), (28), (30) in (18), we get

$$
R_{d}(0, z)=\frac{\lambda z k-\lambda k}{z-(1-c) \overline{F_{1}}(h)-(1-p) c \overline{F_{1}}(h) \overline{F_{2}}(h)-(1-q) p c \overline{F_{1}}(h) \overline{F_{2}}(h) \overline{F_{3}}(h)}
$$

Using (31) in (23), (25), (27), (29) we get

$$
R_{d}(z)=\frac{\left[\overline{F_{1}}(h)-1\right]}{z-(1-c) \overline{F_{1}}(h)-(1-p) c \overline{F_{1}}(h) \overline{F_{2}}(h)-(1-q) p c \overline{F_{1}}(h) \overline{F_{2}}(h) \overline{F_{3}}(h)}
$$$$
S_{d}(z)=\frac{c \overline{F_{1}}(h)\left[\overline{F_{2}}(h)-1\right]}{z-(1-c) \overline{F_{1}}(h)-(1-p) c \bar{F}_{1}(h) \bar{F}_{2}(h)-(1-q) p}
$$

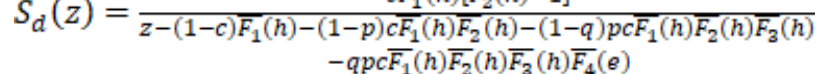

$$
L_{d}^{(1)}(z)=\frac{p c \overline{F_{1}}(h) \overline{F_{2}}(h)\left[\overline{F_{3}}(h)-1\right]}{z-(1-c) \overline{F_{1}}(h)-(1-p) \bar{c} \overline{1_{1}}(h) \overline{F_{2}}(h)-(1-q) p c \overline{F_{1}}(h) \overline{F_{2}}(h) \overline{F_{3}}(h)}
$$

\section{LIKELIHOOD CREATING CAPACITY OF THE LINE ESTIMATE}

Let $M_{d}(z)$ be the probability generating function of the queue size, It is given by

$$
M_{d}(z)=k\left[R_{d}(z)+S_{d}(z)+L_{d}^{(1)}(z)+L_{d}^{(2)}(z)\right]
$$

Adding (32) to (35), we get,

$$
\begin{gathered}
M_{d}(z)=\frac{\left.p c \overline{F_{1}}(h) \overline{F_{2}}(h) \overline{F_{3}}(h)-1\right]+q p c \overline{F_{1}}(h) \overline{F_{2}}(h) \overline{F_{3}}(h) \frac{\left[\overline{F_{4}}(e)-1\right]}{\beta}}{z-(1-c) \overline{F_{1}}(h)-(1-p) c \overline{F_{1}}(h) \overline{F_{2}}(h)-} \\
(1-q) p c \overline{F_{1}}(h) \overline{F_{2}}(h) \overline{F_{3}}(h) \\
-q p c \overline{F_{1}}(h) \overline{F_{2}}(h) \overline{F_{3}}(h) \overline{F_{4}}(e)
\end{gathered}
$$

\section{IDLE TIME AND UTILIZATION FACTOR}

The normalization condition $M_{d}(1)+k=1$

is used to determine k. At $z=1 M_{d}(z)$ attains $0 / 0$ indeterminate form.

Hence using L.H rule we get, $\lim _{z \rightarrow 1} M_{d}(1)=\frac{N^{t}(1)}{D^{t}(1)}$

Hence the utilization factor $\rho$ is found using $\rho=1-k$

To find $L_{q}$ the length of the Queue and the Queue performance measures.

The Average queue size is obtained by the usage of $\mathrm{L} \mathrm{H}$ rule in (36)

$$
\begin{aligned}
& L_{q}(z)=\lim _{z \rightarrow 1} \frac{D^{\prime}(z) N^{n}(z)-N^{\prime}(z) D^{n}(z)}{2\left(D^{\prime}(z)\right)^{2}} \\
& N^{\prime}(1)=\lambda^{r} E\left(F_{1}\right)+c \lambda^{r} E\left(F_{2}\right)+p c \lambda^{r} E\left(F_{3}\right)+\lambda^{r} q p c E\left(F_{4}\right) \\
& N^{\prime \prime}(1)=\left(\lambda^{r}\right)^{2} E\left({F_{1}}^{2}\right)+c\left(\lambda^{r}\right)^{2}\left[2 E\left(F_{1}\right) E\left(E_{2}\right)+E\left(F_{2}{ }^{2}\right)\right]+ \\
& p c\left(\lambda^{r}\right)^{2}\left[2 E\left(F_{1}\right) E\left(F_{3}\right)+2 E\left(F_{2}\right) E\left(F_{3}\right)^{n+e} E^{2}\left(F_{2}\right)\right]+ \\
& q p c\left(\lambda^{r}\right)^{2}\left[2\left(E\left(F_{1}\right)+E\left(F_{2}\right)+F\left(F^{2}\right) E\left(F_{4}\right)+E\left(F_{4}{ }^{2}\right)\right]\right.
\end{aligned}
$$




$$
\begin{aligned}
& D^{\prime}(1)=1-\lambda^{r}(1-c) E\left(F_{1}\right)-(1-p) c \lambda^{r}\left[E\left(F_{1}\right)+\right. \\
& \left.E\left(F_{2}\right)\right]-(1-q) p c \lambda^{r}\left[E\left(F_{1}\right)+E\left(F_{2}\right)+E\left(F_{3}\right)\right]- \\
& q p c \lambda^{r}\left[E\left(F_{1}\right)+E\left(F_{2}\right)+E\left(F_{3}\right)+\beta E\left(F_{4}\right)\right]
\end{aligned}
$$$$
D^{\prime \prime}(1)=-(1-c)\left(\lambda^{r}\right)^{2} E\left({F_{1}}^{2}\right)-(1-P) c\left(\lambda^{r}\right)^{2}\left[E\left({F_{1}}^{2}\right)+\right.
$$$$
\left.E\left(F_{2}^{2}\right)+2 E\left(F_{1}\right) E\left(F_{2}\right)\right]-(1-q) p c\left(\lambda^{r}\right)^{2}\left[E\left(F_{1}^{2}\right)+\right.
$$$$
E\left(F_{2}{ }^{2}\right)+E\left({F_{3}}^{2}\right)+2\left[E\left(F_{1}\right) E\left(F_{2}\right)+E\left(F_{2}\right) E\left(F_{3}\right)+\right.
$$$$
\left.\left.E\left(F_{1}\right) E\left(F_{3}\right)\right]\right]-q p c\left(\lambda^{r}\right)^{2}\left[E\left({F_{1}}^{2}\right)+E\left({F_{2}}^{2}\right)+E\left({F_{3}}^{2}\right)+\right.
$$$$
\beta^{2} E\left(F_{4}^{2}\right)+
$$$$
2\left[E\left(F_{1}\right) E\left(F_{2}\right)+E\left(F_{2}\right) E\left(F_{3}\right)+E\left(F_{3}\right) \beta E\left(F_{4}\right)+\right.
$$$$
\left.\left.\beta E\left(F_{4}\right) E\left(F_{1}\right)+E\left(F_{1}\right) E\left(F_{3}\right)+\beta E\left(F_{2}\right) E\left(F_{4}\right)\right]\right]
$$

Substituting (38) in (37) we obtain $L_{q}$ in closed form.

Further, by the usage of Little's law we get the other execution measures $W_{q}=\frac{L_{q}}{\lambda}, W=\frac{L}{\lambda}, L=L_{q}+\rho$

\section{NUMERICAL ILLUSTRATION}

THE

$$
\text { VALUES }
$$

ARE

COLLECTED

$\lambda^{r}=3, \beta=0.6, c=0.5, p=0.3, q=0.4, \gamma_{r}=3.5, \gamma_{s}=$ $5, \gamma_{l_{1}}=4, \gamma_{l_{2}}=4.5, E\left(F_{1}\right)=\frac{1}{\gamma_{r}}, E\left(F_{2}\right)=\frac{1}{y_{s}}, E\left(F_{3}\right)=$ $\frac{1}{\gamma_{l_{1}}}, E\left(F_{4}\right)=\frac{1}{\gamma_{l_{2}}}, E\left({F_{1}}^{2}\right)=\frac{2}{\gamma_{r}^{2}}, E\left(F_{2}{ }^{2}\right)=\frac{2_{s}}{\gamma_{s}{ }^{2}}, E\left(F_{3}{ }^{2}\right)=$ $\frac{\frac{2}{\gamma_{l_{1}}}}{\gamma^{2}}, E\left({F_{4}}^{2}\right)=\frac{2}{\gamma_{l_{2}}{ }^{2}}$

Table I

Effect of change of( $(\mathrm{p}=0.3,0.4,0.5,0.6,0.7)$

\begin{tabular}{|l|l|l|l|l|l|}
\hline $\mathrm{Q}$ & $\rho$ & $L_{q}$ & $L$ & $W_{\text {q }}$ & $W$ \\
\hline 0.2891 & 0.7109 & 16.7738 & 17.4847 & 5.5913 & 5.8282 \\
\hline 0.3322 & 0.6678 & 13.3433 & 14.0111 & 4.4478 & 4.6704 \\
\hline 0.3747 & 0.6203 & 10.9610 & 11.5813 & 3.6537 & 3.8604 \\
\hline 0.4168 & 0.5832 & 9.2299 & 9.8131 & 3.0766 & 3.2710 \\
\hline 0.4585 & 0.5415 & 7.9177 & 8.4592 & 2.6392 & 2.8197 \\
\hline
\end{tabular}

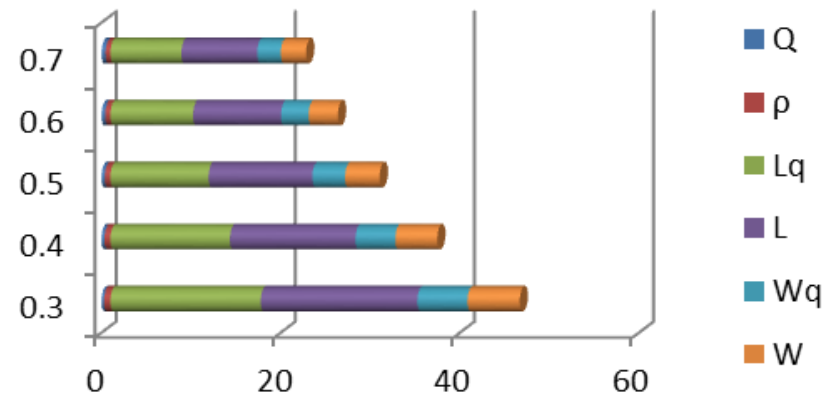

Fig. 1 Effect of change of ${ }^{p}$

From Table I It is clear that if the probability of taking long vacation increases it leads to decrease in all the performance measures. Since the long vacation gets increased the idle time gets amplified.
ACCORDINGLY:

Table II

Effect of change of $(q=0.4,0.5,0.6,0.7,0.8)$

\begin{tabular}{|l|l|l|l|l|l|}
\hline $\mathrm{Q}$ & $\rho$ & $L_{q}$ & $L$ & $W_{q}$ & $W$ \\
\hline 0.2891 & 0.7109 & 16.7738 & 17.4847 & 5.5913 & 5.8282 \\
\hline 0.2939 & 0.7061 & 16.1775 & 16.8836 & 5.3925 & 5.6279 \\
\hline 0.2986 & 0.7014 & 15.6126 & 16.3140 & 5.2042 & 5.4380 \\
\hline 0.3032 & 0.6968 & 15.0766 & 15.7734 & 5.0255 & 5.2578 \\
\hline 0.3079 & 0.6921 & 14.5684 & 15.2605 & 4.8561 & 5.0868 \\
\hline
\end{tabular}

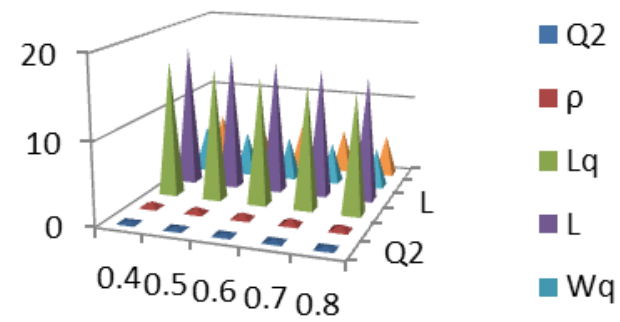

Fig. 2 Effect of change of $q$

Table 2 indicates that, as the probability of taking optional second stage of long vacation gets increased, its corresponding execution measures gets decreased. In addition, idle time increase whereas the utilization factor gets diminished.

\section{CONCLUSION}

The above model gives an intensive report about the lining issue including a few parameters like Single landing, single administration, short excursion, phases of Compulsory long get-away, restricted acceptability during the season of long get-away. The model is very much examined and its practically equivalent to lining execution measures are resolved. Numerical investigation and pictographic portrayal gives an unmistakable image of the model characterized.

\section{REFERENCES}

1. Abdolrahim Badamchi Zadeh, "A Batch Arrival Queue System with Coxian-2 Server Vacations and Admissibility Restricted", American Journal of Industrial and Business Management, Vol 2, pp.47-54, 2012.

2. G. Ayyappan and K. Sathiya , “ $M^{X} / \mathrm{G} / 1$ Feedback Queue with Three Stage Heterogeneous Service and Server Vacations having Restricted Admissibility", Journal of Computations and Modeling, Vol. 3, No. 2, pp. 203-225, 2013.

3. G. Ayyappan and S. Shyamala ,"Time Dependent Solution of $M^{X} / \mathrm{G} / 1$ Queuing Model with second optional service, Bernoulli k- optional vacation and balking", International Journal of Scientific and Research Publications, Vol. 3, No. 9, pp.1-13, 2013.

4. B. Balamurugan and S. Maragathasundari, "A study on the performance analysis of a batch arrival queue with two stages of service Bernoulli schedule vacation extended vacation and service interruption", International journal of computer applications, Vol.124, No.12, pp.33-37, 2015.

5. Dhanalakshmi and S. Maragathasundari ,"Mobile adhoc networks problem- A queuing approach", International journal of communication networks and distributed systems, vol..21, No.4, 2018 . 
6. Kailash C. Madan and Gautam Choudhury, "An $M^{X} / \mathrm{G} / 1$ Queue with a Bernoulli vacation schedule under restricted admissibility policy", The Indian Journal of Statistics, Vol. 66, No. 1. pp. 175-193, 2004.

7. S. Maragathasundari and S. Srinivasan, "Analysis of $\mathrm{M} / \mathrm{G} / 1$ feedback queue with three stage and multiple server vacation", Applied mathematical sciences, Vol..6, No.125, pp.6221-6240, 2012.

8. S. Maragathasundari and S. Srinivasan , "A Non-Markovian Multistage Batch arrival queue with breakdown and reneging", Mathematical problems in engineering, Volume 2014/16 pages/ Article ID 519579/ http: // dx. Doi. Org / 10.1155/2014/ 519579. 2015.

9. Monita Baruah, Kailash C. Madan and Tillal Eldabi , "Balking and Re-service in a vacation Queue with Batch Arrival and Two Types of Heterogeneous Service", Journal of Mathematics Resesarch, Vol. 4, No. 4, pp. 115-124, 2012.

10. Shan Gao and Yunfei Yao, "An $M^{X} / G / 1$ queue with randomized working vacations and at most J vacations", International Journal of Computer Mathematics, Vol. 91, No. 3, pp. 368-383, 2014.

11. S. Sowmiah and S. Maragathasundari, "A study on the analysis of performance measure of bulk input queue with $\mathrm{N}$ type of additional optional service, service interruption and deterministic vacation", Imperial Journal of Interdisciplinary Research, Vol.2, No.8, pp.1436-1444, 2016.

12. Vishwa Nath Maurya , Mathematical modeling and performance analysis of single server two-state batch arrivals and batch service markovian queue with multiple vacations", American Journal of Modeling and Optimization, Vol. 2, No. 2, pp. 39-46, 2014.

13. Wojciech M. Kempa, "Some New Results for Departure Process in the $M^{X} / G / 1$ Queuing system with a Single Vacation and Exhaustive Service", Stochastic Analysis and Applications, Vol 28, No. 1, pp.26-43, 2009.

\section{AUTHORS PROFILE}

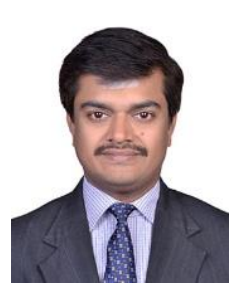

Dr. R. S. Somasundaram is the Professor and Head in the Department of Computer Applications at Coimbatore Institute of Technology, Coimbatore, TamilNadu, India. He received his Ph.D in Computer Science from Bharathiar University, Coimbatore in 2014. He received his Bachelor degree in Mathematics from Madurai Kamaraj University, Madurai in 1993 and Masters degree in Computer Applications from Madurai Kamaraj University, Madurai in 1996. He got qualified as cost accountant in 1996. $\mathrm{He}$ also secured his M.Phil in Computer Science from Bharathiar University in 2005. He has more than 23 years of experience in India and abroad. He has published about 10 research articles in various refereed journals. He is a reviewer of various journals. $\mathrm{He}$ is a member of various professional societies such as ISTE, CSI, IEEE, ACM-CSTA, etc. His research interests includes Queuing theory, Data Mining, Data compression.

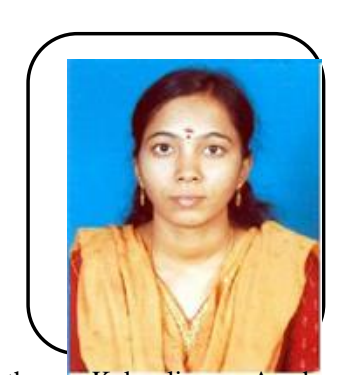

S. Radha pursued her B.Sc degree from Manonmaniam Sundaranar University, Tirunelveli in 2006. She got her B.Ed degree from V.P.M.M. College of Education, Srivilliputur in 2007 and her M.Sc. degree from Annamalai University, Chidambaram in 2009. She obtained her M.Ed. degree from St. Justin's College Of Education , Madurai in 2010. She had 4 years of teaching experience. Now, She is doing research in the area of Queuing theory, Kalasalingam Academy Of Research And Education, Anand Nagar, Tamilnadu, Krishnankovil-626126, India.

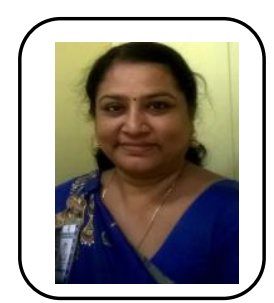

Dr. S. Maragatha Sundari acquired her B.Ed. degree from V.O.C Teachers College, Tuticorin in 1993. She got her M.Sc. in Mathematics from Manonmaniam Sundaranar University; Tirunelveli in 1995 and her M.Phil. degree from Madurai Kamaraj University, Madurai in 2003. She did her $\mathrm{Ph} . \mathrm{D}$. in Sathyabama University, Chennai, India. She has more than 17 years of instructing knowledge. She has distributed more than 50 inquire about papers in national and international journals. She has displayed and distributed papers at national and international conferences. She is right now filling in as an Associate Professor in the Department of Mathematics in Kalasalingam Academy Of Research And Education, Anand Nagar, Tamilnadu, Krishnankovil-626126, India. In addition, she is doing her research guidance for five research scholars. 